\title{
Hyper-cross-linked thin polydimethylsiloxane films
}

\author{
Evelien Maaskant $^{\mathrm{a}}$, Kristianne Tempelman ${ }^{\mathrm{a}}$, Nieck E. Benes ${ }^{\mathrm{a}, *}$ \\ ${ }^{a}$ Films in Fluids, University of Twente, Department of Science and Technology, MESA+ Institute for Nanotechnology, P.O. Box 217, 7500 AE \\ Enschede, The Netherlands
}

\begin{abstract}
Polydimethylsiloxane (PDMS) elastomers are widely used in many applications, such as in microfluidic devices or as membranes. These slightly cross-linked PDMS networks suffer from severe swelling upon contact with, especially non-polar, organic solvents, thereby drastically reducing the performance of the device. Here, we report on a new method to prepare ultra-thin, highly cross-linked PDMS films in a localized fashion by the interfacial polymerization of PDMS with polyhedral oligomeric silsesquioxane (POSS). The resulting thin poly(PDMS-POSSimide) films ( $\sim 150$ $\mathrm{nm}$ ) showed to have a drastic reduction in swelling upon contact with $n$-hexane vapor ( $\sim 15$ fold decrease) and ethyl acetate vapor ( $\sim 5$ fold decrease). These poly(PDMS-POSSimide) films showed an increase in swelling upon contact with ethanol vapor (up to a 4 fold increase) as compared to conventional PDMS. We attribute this increase in swelling to the enhanced affinity for polar solvents of these poly(PDMS-POSSimide) films due to the presence of positively charged ammonium groups. Despite the highest swelling in ethanol, the poly(PDMS-POSSimide) films are found to have extremely low ethanol permeances $\left(<0.1 \mathrm{~L} \mathrm{~m}^{-2} \mathrm{~h}^{-1} \mathrm{bar}^{-1}\right)$, thereby making them potential barrier materials.
\end{abstract}

Keywords: PDMS, POSS, interfacial polymerization, hybrid materials, thin films

\section{Introduction}

Polydimethylsiloxane (PDMS) is an organosilicon polymer, that has the general formula $\left[\mathrm{Si}\left(\mathrm{CH}_{3}\right)_{2} \mathrm{O}\right]_{\mathrm{n}}$. PDMS is widely used because it is optically transparent, flexible, inert, bio-compatible, and non-flammable. Most applications require the cross-linking of PDMS chains. Typically, cross-linked PDMS is prepared from the hydrosilylation of a liquid divinyl-terminated PDMS, with a multifunctional organosilane cross-linker, followed by a (thermal) curing step [1]. The reaction rate of this cross-linking reaction at ambient conditions is sufficiently low to allow for processing the PDMS by methods such as spin-coating, molding, or casting.

Example application areas of PDMS are sensors, medical devices, microfluidics, or membranes [2, 3]. In microfluidic devices, thin PDMS films are commonly applied as, e.g., microlenses [4]-6], valves [7, 8], or actuators [9, 10]. In addition, thin PDMS films are used as membranes in organic solvent nanofiltration (OSN), or as gas separation membranes [11].

*Corresponding author. n.e.benes@utwente.nl 
Although PDMS is widely used in many applications, it has some major drawbacks in the presence of organic solvents. The high affinity of PDMS for non-polar solvents and the rubbery nature of PDMS, causes PDMS to swell substantially, especially in non-polar solvents. This severe swelling could significantly reduce the performance of any PDMS-based device. For example, PDMS membranes can suffer from the loss of selectivity in organic solvents since high molecular weight solutes can permeate through the swollen membrane. In an extreme case, the selective PDMS layer can be peeled off from the support due to severe swelling of the PDMS.

It is for the above mentioned reasons, often desirable to reduce the swelling of PDMS in organic solvents. Several approaches focusing on the reduction of swelling in membranes can be found in literature. These methods could also be applied to other PDMS-based devices. One of these methods is the inclusion of inorganic nanofillers in the PDMS matrix, e.g. silica particles [12, 13], zeolites [14], carbon nanotubes [15], or carbon nanoparticles [16]. These nanofillers physically interact with the PDMS matrix, thereby restricting the swelling of PDMS [11]. However, the use of inorganic nanofillers can result in phase separation, or particle agglomeration, which can reduce the performance of the device [17].

An improved control over the swelling of the PDMS network could be obtained when the nanofiller is covalently bound to the PDMS matrix. An interesting nanofiller that could be covalently cross-linked to PDMS is polyhedral oligomeric silsesquioxane (POSS). Typical POSS molecules have a cage structure with the general formula $\mathrm{R}_{8} \mathrm{Si}_{8} \mathrm{O}_{12}$, where the organic end-groups R can be tailored [18]. POSS is of high interest due to its excellent thermal and chemical stability of the inorganic core, and flexible end-group chemistry. Many examples of the cross-linking of PDMS with partially condensed [19, 20] or fully condensed [20,-24] POSS cages can be found in literature. These PDMS-POSS nanocomposites are even used as membranes for gas separation, although the preparation of these membranes by casting [25] results in relatively thick (200-250 $\mu \mathrm{m})$ PDMS layers [20].

Here, we show the preparation of ultra-thin $(\sim 150 \mathrm{~nm})$ hyper-cross-linked PDMS-POSS films by interfacial polymerization (IP). IP is the localized polycondensation of two or more monomers at the interface of two immiscible solvents [11, 26, 27] resulting in a perfectly alternating cross-linked polymeric film. POSS has already been successfully used in the preparation of hybrid polyamide [28, 29], or polyimide [30] films, prepared from POSS and small organic monomers. However, POSS has not yet been applied in the preparation of cross-linked PDMS films by interfacial polymerization.

To prepare these cross-linked PDMS films, octaAmmonium-POSS was deprotonated to form its reactive free amine, and subsequently reacted with succinic anhydride terminated PDMS resulting in a poly(PDMS-POSSamic acid). This poly(amic acid) was thermally imidized in the corresponding poly(PDMS-POSSimide) (Figure 1). The swelling of these poly(PDMS-POSSimide)s was studied by spectroscopic ellipsometry, and compared to the swelling of conventional PDMS. 


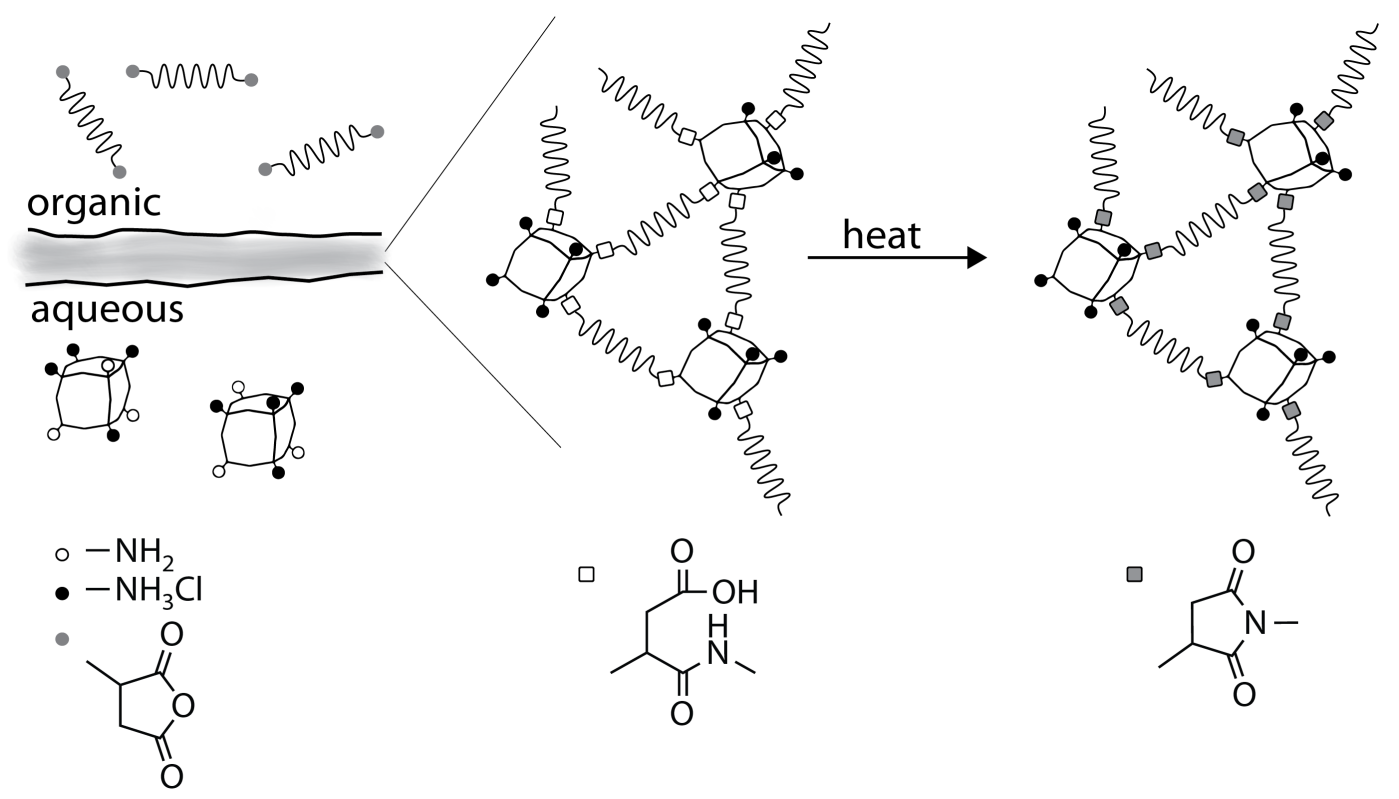

Figure 1: The synthesis of hyper-cross-linked poly(PDMS-POSSimide) by interfacial polymerization of amine functionalized POSS with succinic anhydride functionalized PDMS. After film formation, the resulting poly(PDMS-POSSamic acid) was thermally imidized to the stable poly(PDMSPOSS imide).

\section{Experimental}

\subsection{Materials}

OctaAmmonium POSS (POSS) was obtained from Hybrid Plastics, USA, Polydimethylsiloxane, succinic anhydride terminated; viscosity 75-100 cSt (PDMS) was obtained from ABCR, Germany. Ethanol (absolute) was obtained from Merck. Sodium hydroxide $(\mathrm{NaOH},>98 \%)$, toluene (anhydrous, 99.8\%), and ethyl acetate (anhydrous, 99.8\%) were obtained from Sigma-Aldrich (The Netherlands). PDMS, Permacol RTV 615 A/B type was obtained from Permacol B.V. as a two component system, consisting of a vinyl terminated siloxane polymer and cross-linker (RTV A) and a polydimethyl hydrogen siloxane with a catalyst (RTV B).

\subsection{Preparation of PDMS films}

Thin PDMS films were prepared on silicon wafers by spin-coating a pre-polymer solution ( $2.5 \mathrm{wt} \%$ in $n$-hexane) containing the cross-linker in a ratio of RTV A/RTV B of 10:1 at $3000 \mathrm{rpm}$ for 30 seconds. To facilitate the crosslinking, the thin films were were placed in a chamber oven (Carbolite HTMA 5/28) under a nitrogen flow (5 mL $\left.\min ^{-1}\right)$. The samples were heated to $80^{\circ} \mathrm{C}$ for $8 \mathrm{~h}$.

\subsection{Synthesis of poly(PDMS-POSSamic acid) networks by interfacial polymerization}

Both powdered and supported poly(PDMS-POSSamic acid) networks were prepared by interfacial polymerization (IP). For both, POSS was dissolved in Milli-Q grade water, and the $\mathrm{pH}$ of the solution was adjusted to $\mathrm{pH} 8.5 \mathrm{or} 9.5$ 
using 1.5 M NaOH. Powdered poly(PDMS-POSSamic acid) was prepared by vigorously stirring equal amounts of an aqueous solution of POSS ( $0.9 \mathrm{wt} \%)$ and an organic solution of PDMS ( $0.5 \mathrm{wt} \%$ in toluene) for 1 hour. The formed solids were filtered and washed with excess water and toluene, respectively. The precipitate was dried overnight in a vacuum oven at $50{ }^{\circ} \mathrm{C}$, and ground to a fine powder. The powder was stored under nitrogen until further use.

poly(PDMS-POSSamic acid) membranes were prepared by interfacial polymerization on top of porous $\alpha$-alumina disks, coated with two layers of $\gamma$-alumina (Pervatech, The Netherlands). The support was soaked with aqueous POSS solution $(0.9 \mathrm{wt} \%)$ and dried for 15 minutes to remove excess aqueous solution. Afterwards, the organic solution of PDMS ( $0.5 \mathrm{wt} \%$ in toluene) was poured onto the porous disk. After 5 minutes reaction time, the toluene solution was removed, and the membrane surface was washed with toluene.

Thin poly(PDMS-POSSamic acid) films deposited on silicon wafers were prepared for spectroscopic ellipsometry experiments. A silicon wafer was fixed in the middle of a petri dish and aqueous POSS solution ( $0.9 \mathrm{wt} \%, \mathrm{pH}=9.5)$ was poured into the petri dish, just covering the wafer. Then PDMS solution ( $0.5 \mathrm{wt} \%$ in toluene) was gently poured from the side over the POSS solution, allowing it to float on top of the aqueous solution. A thin film formed immediately. After ten minutes reaction time, most of the toluene was removed with a pipet and the petri dish was slowly decanted, allowing for the water solution to flow out of the petri dish, and the formed poly(PDMS-POSSamic acid) film was carefully deposited on the fixed wafer. The wafer with the poly(PDMS-POSSamic acid) film was removed from the petri dish and air dried. Once dried, the wafer with the film was carefully placed in a petri dish filled with ethanol, to remove unreacted monomers.

\subsection{Imidization of poly(PDMS-POSSamic acid) networks}

Both poly(PDMS-POSSamic acid) powder and supported films were placed in a chamber oven (Carbolite HTMA $5 / 28)$ under a nitrogen flow $\left(5 \mathrm{~mL} \mathrm{~min}^{-1}\right)$. The samples were heated to $100{ }^{\circ} \mathrm{C}$ for $1 \mathrm{~h}$, followed by a 18 minutes dwell at $250{ }^{\circ} \mathrm{C}$ with a heating rate of $10^{\circ} \mathrm{C} \mathrm{min}^{-1}$. The imidization time and temperature were determined from combined TGA-MS experiments. The samples were cooled to room temperature without a specified cooling rate.

\subsection{Characterization}

The chemical structure of poly(PDMS-POSSamic acid) and poly(PDMS-POSSimide) powders was analyzed by fourier transform infrared spectroscopy (FTIR) in attenuated total reflectance (ATR) mode on an ALPHA spectrometer (Bruker, Germany). All powders were used without further sample preparation.

Combined thermogravimetric analysis (TGA) and mass spectroscopy (MS) measurements were performed on poly(PDMS-POSSamic acid) powders using an STA 449 F3 Jupiter TGA with an aluminum sample cup (Netzsch, Germany) and QMS 403 D Aeolos MS (Netzsch, Germany). Mass loss measurements were performed from 50 to $1000{ }^{\circ} \mathrm{C}$ with a heating rate of $20{ }^{\circ} \mathrm{C} \mathrm{min}^{-1}$ under both air and nitrogen atmospheres. Isothermal analysis was performed at $250{ }^{\circ} \mathrm{C}$ with a heating rate of $10^{\circ} \mathrm{C} \mathrm{min}^{-1}$ under nitrogen atmosphere. 
Streaming current measurements were performed on a SurPASS (Anton Paar, Austria) analyzer equipped with a adjustable gap cell. Poly(PDMS-POSSimide) films were deposited on silicon wafers of $1 \times 2 \mathrm{~cm}^{2}$. The wafers were attached to the adjustable gap cell by double sided adhesive tape. The $\mathrm{pH}$ of the electrolyte solution $(5 \mathrm{mM} \mathrm{KCl})$ was automatically adjusted using $0.1 \mathrm{M} \mathrm{NaOH}$. From the streaming current, the zeta potential could be derived using Equation 1,

$$
\zeta=\frac{d I}{d P} \cdot \frac{\eta}{\epsilon \cdot \epsilon_{0}} \cdot \frac{L}{A_{\text {channel }}}
$$

where $\zeta$ is the zeta potential, $\frac{\mathrm{d} I}{\mathrm{~d} P}$ is the slope of the streaming current versus pressure, $\eta$ and $\epsilon$ are the viscosity and dielectric constant of the electrolyte, here taken as that of water, $\epsilon_{0}$ is the permittivity of vacuum, $L$ is the length of the streaming channel, and $A_{\text {channel }}$ is the cross sectional area of the channel.

The contact angle of poly(PDMS-POSSimide) films deposited on silicon wafers was measured with an OKA 15 (DataPhysics, Germany) set-up by applying the sessile drop method. A Milli-Q water droplet $(V=1 \mu \mathrm{L})$ was deposited onto the surface and the contact angle was obtained $3 \mathrm{~s}$ after deposition. The average contact angle and $95 \%$ confidence interval were calculated from 5 individual measurements.

Ethanol permeation measurements were performed on a custom-built dead-end permeation setup. A feed vessel was pressurized to 20 bar using ultra pure $\mathrm{N}_{2}$. Permeate streams were measured with a mass balance.

\subsection{In-situ spectroscopic ellipsometry}

Thickness and refractive index measurements of films were done with spectroscopic ellipsometry on an M2000X spectroscopic ellipsometer (J.A. Woollam Co.), with focussing probes. In-situ data was recorded at least once every 15 seconds. The data was modelled using the CompleteEase software package (J.A. Woollam Co.) in the wavelength range of 370-1000 $\mathrm{nm}$. The substrate was modelled using the built-in temperature-dependent optical properties of silicon, on top of which a $2 \mathrm{~nm}$ native oxide layer was modelled. The refractive index of the poly(PDMS-POSS) film was modelled at $632.8 \mathrm{~nm}$ wavelength using a Cauchy dispersion (fit parameters: $A, B, k$, and thickness).

The ellipsometry data for imidization was collected with a Linkam heating stage with quartz windows with an incident angle of $70^{\circ}$. The non-imidized films, used for imidization measurements with ellipsometry, were stripped of residual solvents in a furnace at $110{ }^{\circ} \mathrm{C}$ for 20 minutes. The temperature measurements were performed under ultra pure $\mathrm{N}_{2}$ at a flow rate of $100 \mathrm{~mL} \mathrm{~min}^{-1}$. A temperature correction was performed according to the procedure described by Kappert et al. [31] (the difference between set point and actual temperature was less than $1{ }^{\circ} \mathrm{C}$ ). The optical retardance of the windows of the measurement cell was included using calibrated delta off-sets. Prior to heating, the samples were flushed at $25{ }^{\circ} \mathrm{C}$ under $\mathrm{N}_{2}$ flow for 30 minutes. Then, the samples were heated to $250{ }^{\circ} \mathrm{C}$ at $5{ }^{\circ} \mathrm{C}$ min ${ }^{-1}$ and remained at $250{ }^{\circ} \mathrm{C}$ for 20 minutes. The samples were cooled down with a cooling rate of $20{ }^{\circ} \mathrm{C} \mathrm{min}^{-1}$.

The obtained thickness was divided by the thickness measured at time $t=0$ resulting in the relative thickness as shown by Equation 2

$$
d_{\text {relative }}=\frac{d_{t}}{d_{0}}
$$


Where $d_{\text {relative }}$ is the relative thickness at a certain point of time, and $d_{0}$ and $d_{t}$ are the thicknesses at the starting time or at a certain point of time, respectively. The relative refractive index was obtained similarly from the refractive index at the starting time and at a certain point of time.

The ellipsometry swelling measurements were performed with a custom-built Teflon liquid flow cell with quartz windows with an incident angle of $70^{\circ}$. The cell volume was $25.5 \mathrm{~cm}^{3}$. The cell temperature was controlled with an external water bath (LAUDA-Brinkmann, LP.), allowing water at $25{ }^{\circ} \mathrm{C}$ to flow through the outer walls of the cell. The optical retardance of the windows of the measurement cell was corrected for using calibrated delta offsets. The solvent vapor flow was generated using two identical bubblers in series, both at $25^{\circ} \mathrm{C}$. The carrier gas was a ultra pure $\mathrm{N}_{2}$ gas, with a gas flow of $100 \mathrm{~mL} \mathrm{~min}^{-1}$. The dry sample thickness and refractive index were based on the last ten data points before the solvent vapor was introduced into the cell.

The poly(PDMS-POSSimide) films show a significant spread in the refractive index, that can originate from the preparation of the films on a silicon wafer. The preparation involves a free standing film that is deposited onto the silicon wafer by decanting the solutions. As a result, an inhomogeneous thin film was deposited onto the wafer, with different thicknesses and refractive indices, depending on the local reaction time. As a result of the inhomogeneity of the poly(PDMS-POSSimide) films, the refractive index is considered to be less reliable. The behaviour of the refractive index is, however, given in the supplementary information.

\section{Results and discussion}

\subsection{Imidization of poly(PDMS-POSSamic acid)}

The imidization of poly(PDMS-POSSamic acid) to poly(PDMS-POSSimide) is an important step to ensure stability of the material against hydrolysis. Poly(PDMS-POSSamic acid) powder has been thermally treated during a thermogravimetric analysis combined with mass spectroscopy (TGA-MS) to study the onset of imidization and the stability of the material during thermal treatment. Figure 2 shows the mass loss as function of temperature (upper panel) and the corresponding gases evolved (lower panel). During imidization, the amic acid ring is closed while water is eliminated (Figure 1). Thus the onset of imidization can be determined from the $m / z=18$ MS signal.

From Figure 2 it can be concluded that the imidization of this poly(PDMS-POSSamic acid) starts at $150{ }^{\circ} \mathrm{C}$, with a peak in water release at $195{ }^{\circ} \mathrm{C}$. Above $250{ }^{\circ} \mathrm{C}$ no more water is released, indicating that the imidization is finished at this temperature. The most significant mass loss and the release of decomposition products (e.g. $\mathrm{CO}_{2}, \mathrm{CH}_{4}$, and $\mathrm{NO}$ ) starts at temperatures above $400{ }^{\circ} \mathrm{C}$, indicating the excellent thermal stability of the poly(PDMS-POSSimide) material. Infrared spectroscopy measurements (see Figure S2 in Supplementary Information) confirmed the conversion of the covalent amic acid bonds to imide bonds between the PDMS and POSS after the thermal treatment. 

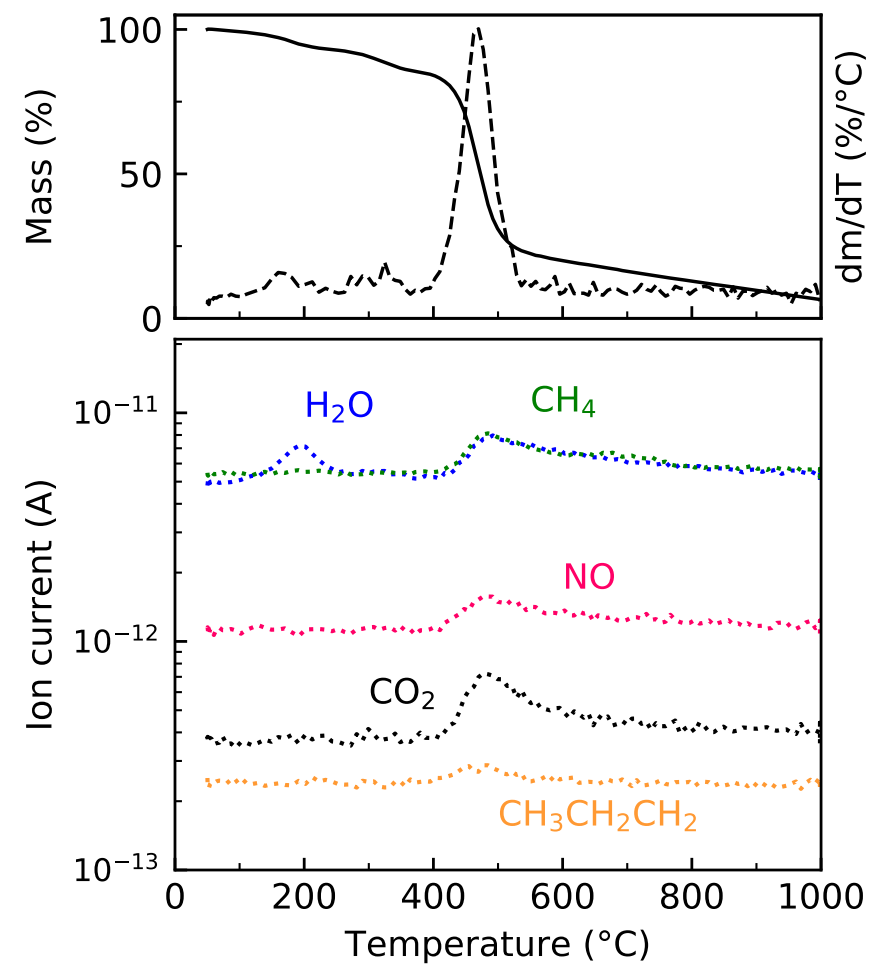

Figure 2: Combined TGA-MS analysis under nitrogen atmosphere of poly(PDMS-POSSamic acid) powder. The upper panel shows the mass loss as function of temperature. The lower panel shows the evolved gases during heating. The following gases are traced: $\mathrm{CH}_{4}(\mathrm{~m} / z=15$, green, - $)$, $\mathrm{H}_{2} \mathrm{O}(\mathrm{m} / z=18$, blue, 一 $), \mathrm{NO}\left(\mathrm{m} / \mathrm{z}=30\right.$, pink, 一), $\mathrm{CH}_{3} \mathrm{CH}_{2} \mathrm{CH}_{2}(\mathrm{~m} / \mathrm{z}=43$, orange, -$)$, and $\mathrm{CO}_{2}(\mathrm{~m} / \mathrm{z}=44$, black, 一). 
In addition to the TGA-MS experiments, the imidization process of a thin poly(PDMS-POSSamic acid) film has been studied by spectroscopic ellipsometry. Figure 3 shows the relative thickness $(a, b)$ and relative refractive index $(\mathrm{c}, \mathrm{d})$ as a function of temperature and time. Three regimes can be distinguished when looking at the thickness of the film during the heating phase. First, a small decrease in film thickness is observed at temperatures up to $50{ }^{\circ} \mathrm{C}$. This decrease can be attributed to the drying of the film, and thus the release of physically bound water. The second stage is characterized by a plateau in the film thickness. At this stage the thermal expansion of the film more or less equals the mass loss due to the release of water. However, the refractive index decreases at this stage, indicating a change in chemical composition of the material. The last stage can be defined as the onset of imidization, starting from $180{ }^{\circ} \mathrm{C}$ with a significant decrease in film thickness. This decrease can be attributed to reorganization of the polymer network, while the decrease in index can be attributed to the conversion of the amic acid groups into imide groups [32].

At the isothermal stage (Figure 3 3 ,d) both the thickness and refractive index keep decreasing over time. Since there is no more thermal expansion of the material, the decrease in both thickness and refractive index is attributed to the continuing imidization. During the imidization, progressively less amic acid groups remain, and thereby the decrease in thickness levels off. 

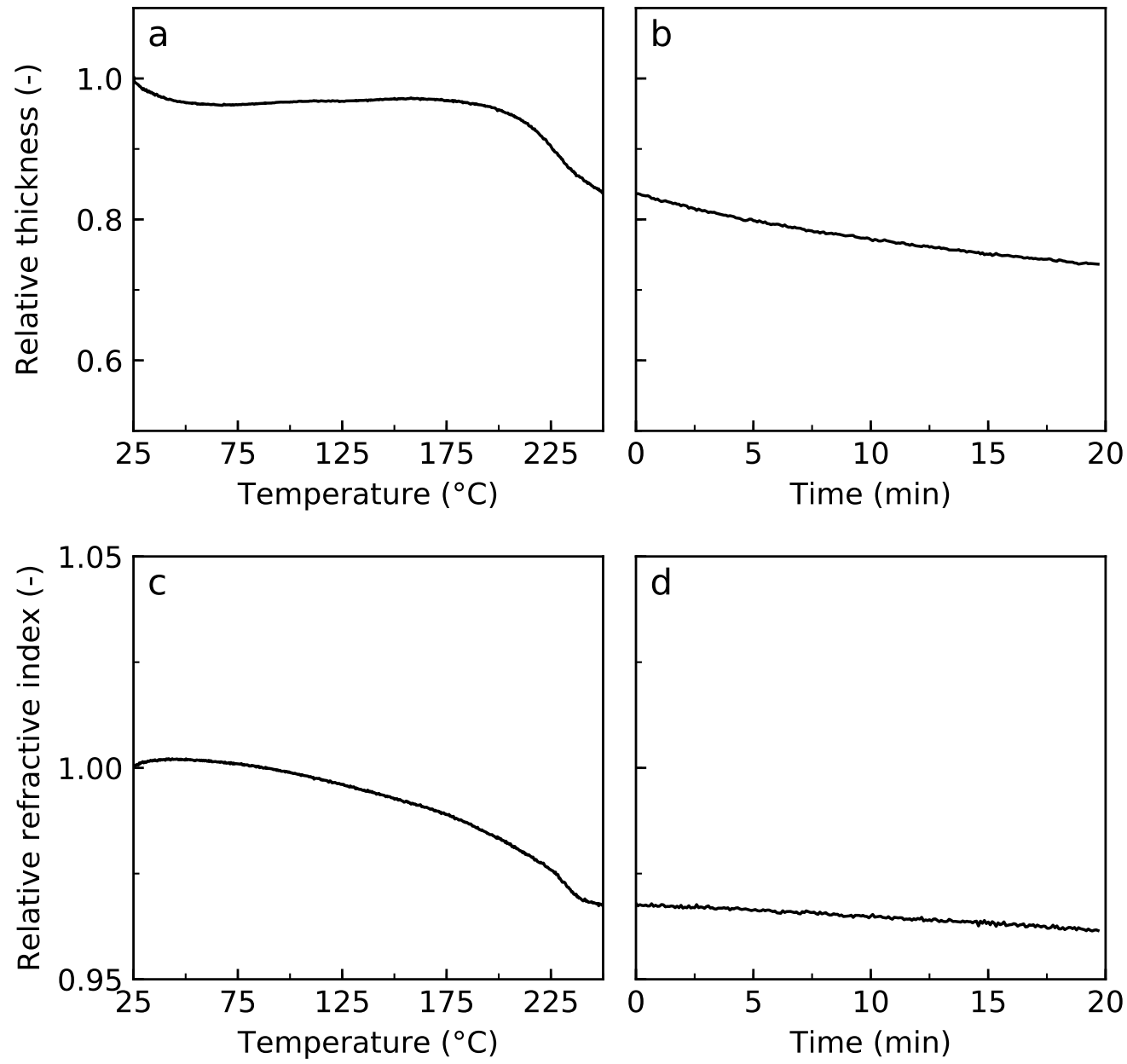

Figure 3: The relative thickness and refractive index of poly(PDMS-POSSamic acid) films during imidization studied by spectroscopic ellipsometry. (a) The relative thickness during heating to $250{ }^{\circ} \mathrm{C}$ as function of temperature. (b) The relative thickness during the isothermal stage as function of time. (c) The relative refractive index during heating to $250{ }^{\circ} \mathrm{C}$ as function of temperature. (d) The relative refractive index during the isothermal stage as function of time.

\subsection{Swelling in organic solvents}

In this work we have studied the swelling of both conventional PDMS films and poly(PDMS-POSSimide) films, in $n$-hexane, ethyl acetate, and ethanol vapors. Figure 4 shows the swelling behavior of a conventional PDMS film as function of the "apparent solvent activity". The extent of solvent induced swelling of a PDMS film is correlated to the affinity of the PDMS chains for the solvent. The conventional PDMS film has the highest swelling degree for $n$-hexane, with a stable value reached at $72 \%$. The second highest swelling degree is observed for ethyl acetate (25\%), and the lowest for ethanol (3\%). This order of the swelling degrees correspond well with the affinity of PDMS for organic solvents, based on Hildebrand solubility parameters (see Table 1), and the shape of the isotherms is consistent with the Flory-Huggins theory. The absolute magnitude of swelling is substantially lower for the swelling 
Table 1: Solubility parameters of the solvents used in this study, the solubility parameter of PDMS is $7.3 \mathrm{cal}^{1 / 2} \mathrm{~cm}^{-3 / 2}[35$.

\begin{tabular}{ll} 
Solvent & $\delta\left(\mathrm{cal}^{1 / 2} \mathrm{~cm}^{-3 / 2}\right)$ \\
\hline$n$-hexane & 7.3 \\
ethyl acetate & 9.0 \\
ethanol & 12.7
\end{tabular}

in vapors than for the pure liquid solvents [33-35]. This indicates that in our experimental method the $\mathrm{N}_{2}$ stream is not completely saturated with the vapor. The use of vapors rather than liquids allows for a higher optical contrast; highly swollen films have a refractive index that is relatively similar to that of the liquid ambient, whereas for vapors the ambient refractive index remains close to unity. A drawback of the use of vapors is that the quantitative control of the activity is generally less accurate, while in particular close to an activity of one the extent of swelling is a strong function of the activity. Moreover, for ethyl acetate the swelling monotonically increases with time over a period of more than an hour. The dynamics of swelling of the rubbery PDMS by organic solvents are very fast as compared to the time-scale of this increase in swelling, indicating that for the ethyl acetate measurement the apparent activity of the vapor in the cell only slowly increases in time.

The poly(PDMS-POSSimide) films show much less swelling as compared to conventional PDMS for $n$-hexane and ethyl acetate (Figure 5a-d). Surprisingly, the most polar solvent ethanol swells the poly(PDMS-POSSimide) films much more that the least polar solvent $n$-hexane (Figure 5-f). In addition, all poly(PDMS-POSSimide) films show a similar trend with respect to the value of the refractive index of the dry films, which is more pronounced with solvent polarity. An increased refractive index indicates an increase of the POSS content inside the poly(PDMS-POSSimide)

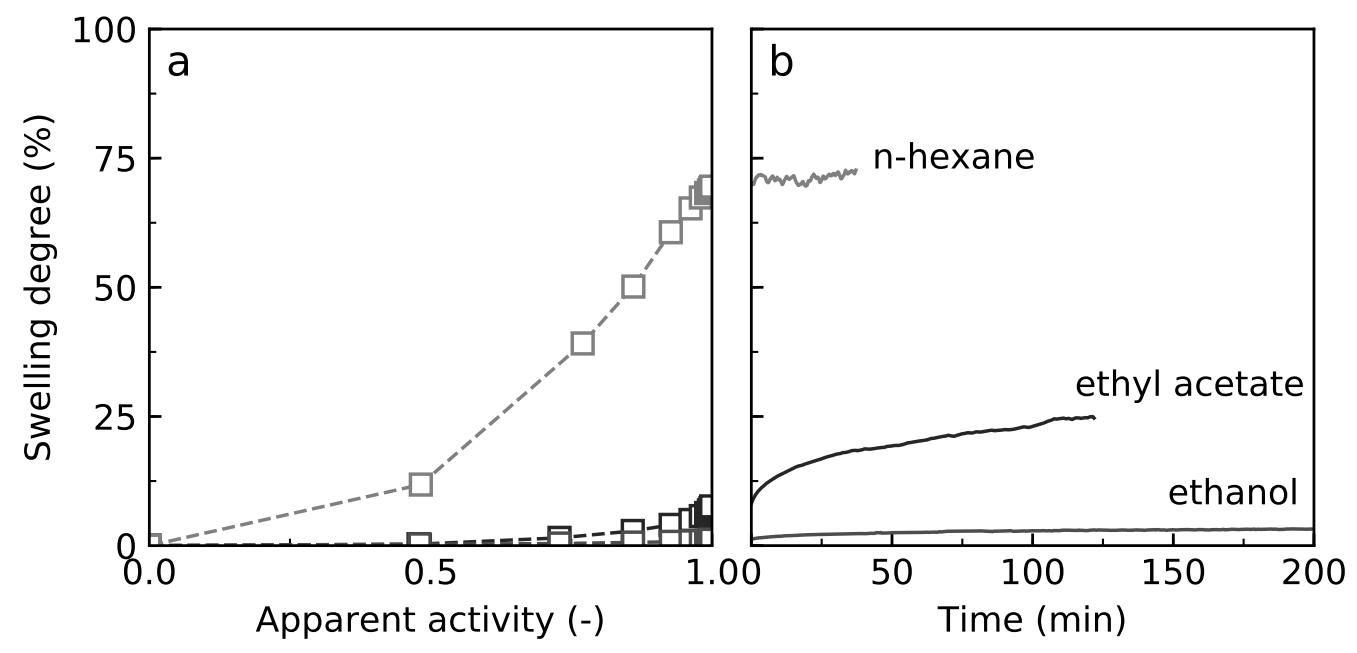

Figure 4: (a) The swelling degree of pure PDMS as a function of solvent vapor activity in the cell. (b) The swelling degree as function of time at an apparent activity of one. The swelling degree of PDMS is the highest for $n$-hexane (72\%), followed by ethyl acetate (25\%), and ethanol (3\%). 
film, as was shown by Raaijmakers et al. [36].

Poly(PDMS-POSSimide)s films show a stable swelling degree of 4-6\% in $n$-hexane, where the swelling is correlated to the refractive index of the film. This swelling degree is approximately fifteen times lower compared to that of the conventional PDMS. This can be caused by the successful cross-linking of the PDMS chains, as well as by the reduced affinity for $n$-hexane due to the inclusion of the POSS molecules.

The swelling degree of poly(PDMS-POSSimide) films in ethyl acetate (3-7\%) is comparable to that obtained for $n$-hexane. This swelling degree is approximately 5 times lower compared to that of conventional PDMS in ethyl acetate. In addition, the correlation of the swelling degree to the refractive index of the poly(PDMS-POSSimide) film is more strongly confirmed as compared to swelling in $n$-hexane.

The effect of refractive index of the poly(PDMS-POSSimide) film on swelling degree is even more pronounced when the films were swollen in ethanol. Where PDMS showed a swelling degree of 3\%, the swelling degree of poly(PDMS-POSSimide) films varied from 4-12\%, with again the highest swelling degree for the lowest refractive index, and thus lowest cross-link density. This higher swelling degree, and more pronounced effect of refractive index can be related to the affinity of the POSS molecules inside the poly(PDMS-POSSimide) film towards ethanol. 

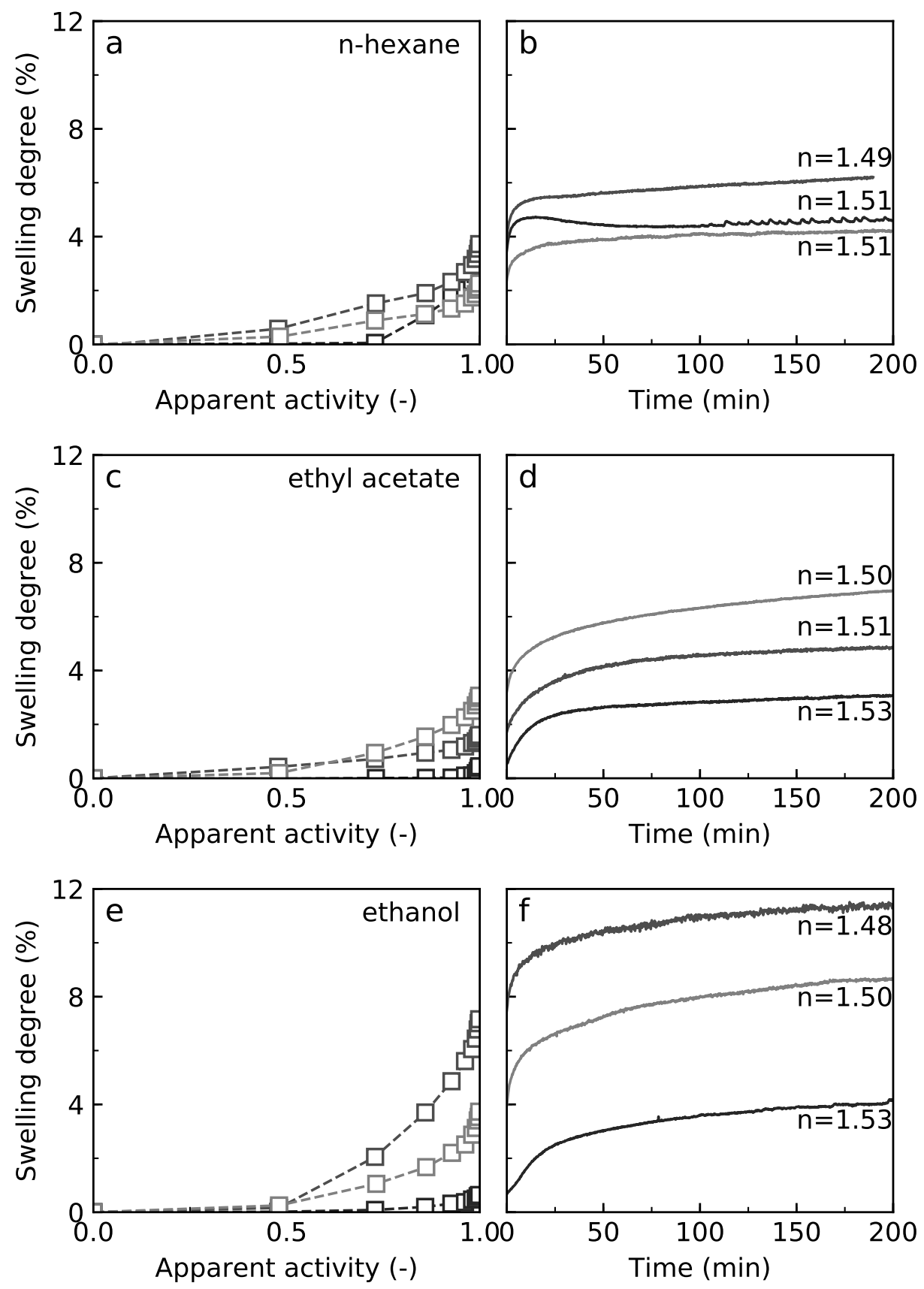

Figure 5: The swelling degree of poly(PDMS-POSSimide) films measured by spectroscopic ellipsometry for (a,b) $n$-hexane, (c,d) ethyl acetate, and (e,f) ethanol vapors. Panels a, c, and e show the swelling degree as function of the apparent solvent vapor activity in the cell. Panels b, d, and f show the swelling degree as function of time at an apparent activity of one. For every solvent, three different samples with varying refractive indices were measured. 


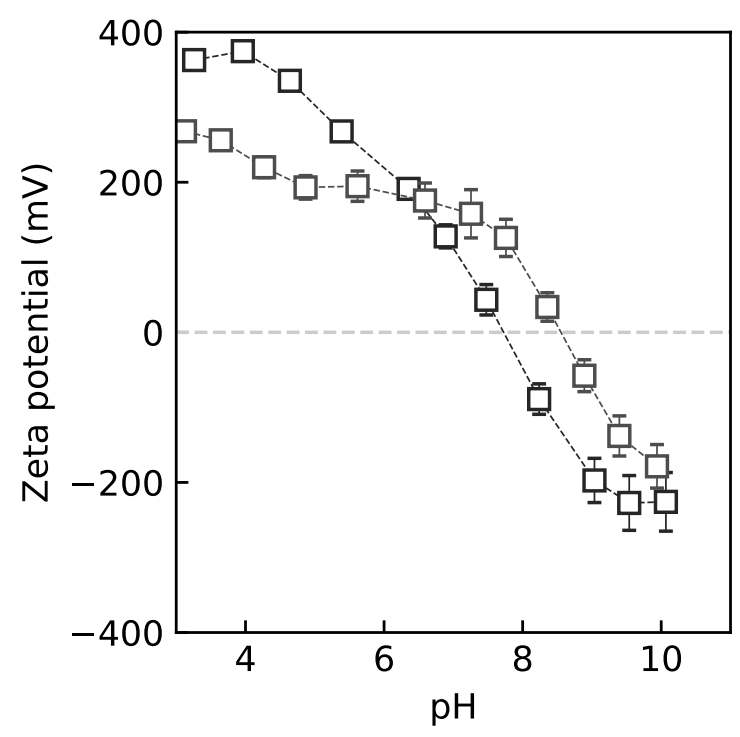

Figure 6: Zeta potential of poly(PDMS-POSSimide) films supported by silicon wafers, measured in duplicate.

The PDMS chains are cross-linked by POSS containing ammonium side-groups. These side-groups are partially deprotonated towards the reactive free amine groups prior to interfacial polymerization. However, only 3-4 ammonium groups are converted at the $\mathrm{pH}$ of the aqueous solution $(\mathrm{pH}=9.5)$ [28], and thus POSS is a positively charged monomer at these conditions. Figure 6 shows the zeta potential of a poly(PDMS-POSSimide) film supported on a silicon wafer, proving the positive charge of the poly(PDMS-POSSimide) film at neutral $\mathrm{pH}$. Therefore, the higher swelling degree of poly(PDMS-POSSimide) films in ethanol could be explained by the presence of positively charged ammonium groups. Despite the positively charged surface, the poly(PDMS-POSSimide) films show to have an average water contact angle of $110^{\circ} \pm 6^{\circ}$ and can therefore be considered as hydrophobic.

\subsection{Barrier properties}

Poly(PDMS-POSSimide) films were prepared by interfacial polymerization on top of $\gamma$-alumina coated porous $\alpha$ alumina supports. The use of these ceramic supports allow for the imidization at $250{ }^{\circ} \mathrm{C}$. Due to the hydrophilic nature of the support, only polar solvents will be permeating through the support. Clean ethanol fluxes were measured for three separate membranes in dead-end mode at 20 bar transmembrane pressure for at least 27 hours. All membranes showed almost no permeation, with ethanol permeances between 0.004 and $0.011 \mathrm{~L} \mathrm{~m}^{-2} \mathrm{~h}^{-1} \mathrm{bar}^{-1}$ (Figure 7), which is surprising regarding the substantial swelling in ethanol observed for films with a lower cross-linking degree. The extremely low ethanol permeance of the poly(PDMS-POSSimide) membrane could be explained by a combination of a high cross-link density, and the relatively short PDMS oligomer used $\left(\mathrm{M}_{\mathrm{n}} \approx 900 \mathrm{~g} \mathrm{~mol}^{-1}\right.$ as determined by ${ }^{1} \mathrm{H}$ NMR). The low permeance of these poly(PDMS-POSSimide) films could be of benefit when applied in microfluidic devices. 


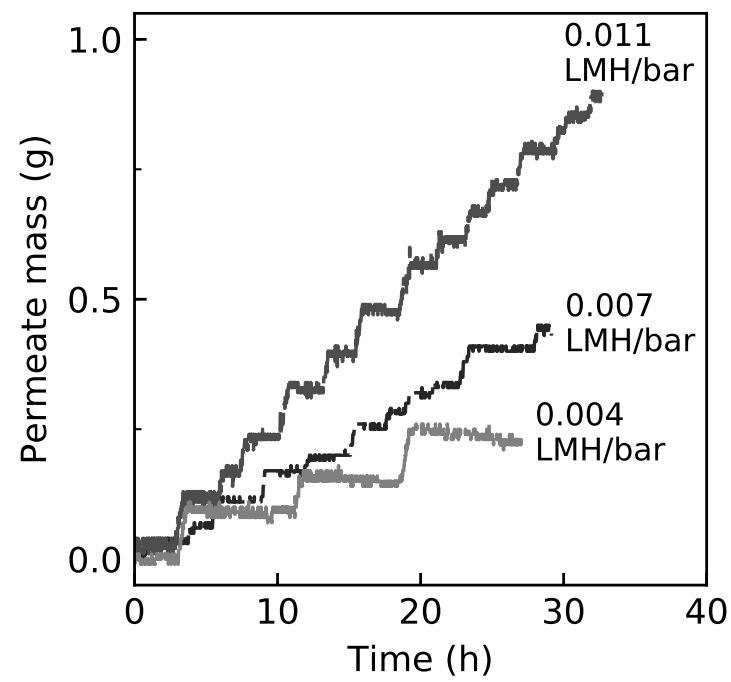

Figure 7: The permeate mass increase during ethanol permeation at 20 bar in dead-end mode. The steps are caused by dripping of the permeate onto the mass balance.

\section{Conclusion}

Here, we showed the preparation of hyper-cross-linked poly(PDMS-POSSimide) films. Swelling measurements in $n$-hexane, ethyl acetate and ethanol vapors show that the presence of the hybrid cross-links restricts the swelling of poly(PDMS-POSSimide) films in $n$-hexane and ethyl acetate significantly as compared to conventional PDMS. A $\sim 15$ fold decrease in swelling in $n$-hexane vapor, and a $\sim 5$ fold decrease in ethyl acetate vapor was observed for these poly(PDMS-POSSimide) films. The presence of unreacted positively charged ammonium groups promote a slightly higher swelling of poly(PDMS-POSSimide) films in ethanol compared to PDMS (similar up to a 4 fold increase). In general, the swelling of these hyper-cross-linked poly(PDMS-POSSimide) films is limited to less than 12\%, showing that these films have only a limited swelling in a wide variety of solvents.

Despite the substantial swelling in ethanol, poly(PDMS-POSSimide) films did not show ethanol permeance $(<0.1$ $\mathrm{L} \mathrm{m}^{-2} \mathrm{~h}^{-1}$ bar $\left.^{-1}\right)$. This low permeance can be attributed to a combination of a high cross-linking degree and the relatively short PDMS oligomer used.

\section{Acknowledgements}

This work took place within the framework of the Institute for Sustainable Process Technology (ISPT, project BL-20-02) and the research programme TA-ISPT Fundamentals with project number 731.014.203, which is (partly) financed by the Netherlands Organisation for Scientific Research (NWO). 


\section{Data availability}

The raw data required to reproduce these findings are available to download from DOI: 10.4121/uuid:7efe302227bf-4a6d-8961-49d48ff31b05. The processed data required to reproduce these findings are available to download from DOI:10.4121/uuid:7efe3022-27bf-4a6d-8961-49d48ff31b05.

\section{References}

[1] A. Esteves, J. Brokken-Zijp, J. Laven, H. Huinink, N. Reuvers, M. Van, G. de With, Influence of cross-linker concentration on the crosslinking of pdms and the network structures formed, Polymer 50 (2009) 3955 - 3966.

[2] S. K. Sia, G. M. Whitesides, Microfluidic devices fabricated in poly(dimethylsiloxane) for biological studies, ELECTROPHORESIS 24 (2003) 3563-3576.

[3] G. Firpo, E. Angeli, L. Repetto, U. Valbusa, Permeability thickness dependence of polydimethylsiloxane (pdms) membranes, Journal of Membrane Science 481 (2015) 1 - 8.

[4] A. Werber, H. Zappe, Tunable microfluidic microlenses, Appl. Opt. 44 (2005) 3238-3245.

[5] T.-K. Shih, C.-F. Chen, J.-R. Ho, F.-T. Chuang, Fabrication of pdms (polydimethylsiloxane) microlens and diffuser using replica molding, Microelectronic Engineering 83 (2006) 2499 - 2503. Materials for Advanced Metallization (MAM 2006).

[6] X. Zeng, H. Jiang, Polydimethylsiloxane microlens arrays fabricated through liquid-phase photopolymerization and molding, Journal of Microelectromechanical Systems 17 (2008) 1210-1217.

[7] M. A. Unger, H.-P. Chou, T. Thorsen, A. Scherer, S. R. Quake, Monolithic microfabricated valves and pumps by multilayer soft lithography, Science 288 (2000) 113-116.

[8] Y. Sugiura, H. Hirama, T. Torii, Fabrication of microfluidic valves using a hydrogel molding method, Scientific Reports 5 (2015) 13375.

[9] F. B. Madsen, A. E. Daugaard, S. Hvilsted, A. L. Skov, The current state of silicone-based dielectric elastomer transducers, Macromolecular Rapid Communications 37 (2016) 378-413.

[10] S. Rosset, H. R. Shea, Flexible and stretchable electrodes for dielectric elastomer actuators, Applied Physics A 110 (2013) $281-307$.

[11] P. Vandezande, L. E. M. Gevers, I. F. J. Vankelecom, Solvent resistant nanofiltration: separating on a molecular level, Chem. Soc. Rev. 37 (2008) 365-405.

[12] K. Vanherck, A. Aerts, J. Martens, I. Vankelecom, Hollow filler based mixed matrix membranes, Chem. Commun. 46 (2010) $2492-2494$.

[13] M. Rezakazemi, A. Vatani, T. Mohammadi, Synergistic interactions between poss and fumed silica and their effect on the properties of crosslinked pdms nanocomposite membranes, RSC Adv. 5 (2015) 82460-82470.

[14] L. E. M. Gevers, I. F. J. Vankelecom, P. A. Jacobs, Zeolite filled polydimethylsiloxane (pdms) as an improved membrane for solvent-resistant nanofiltration (srnf), Chem. Commun. (2005) 2500-2502.

[15] C. Xue, G.-Q. Du, L.-J. Chen, J.-G. Ren, J.-X. Sun, F.-W. Bai, S.-T. Yang, A carbon nanotube filled polydimethylsiloxane hybrid membrane for enhanced butanol recovery, Scientific Reports 4 (2014) 5925 EP -.

[16] H. Azimi, F. H. Tezel, J. Thibault, Effect of embedded activated carbon nanoparticles on the performance of polydimethylsiloxane (pdms) membrane for pervaporation separation of butanol, Journal of Chemical Technology \& Biotechnology 92 (2017) $2901-2911$.

[17] G. Kickelbick, Concepts for the incorporation of inorganic building blocks into organic polymers on a nanoscale, Progress in Polymer Science 28 (2003) $83-114$.

[18] D. B. Cordes, P. D. Lickiss, F. Rataboul, Recent developments in the chemistry of cubic polyhedral oligosilsesquioxanes, Chemical Reviews 110 (2010) 2081-2173. PMID: 20225901

[19] Y. Shi, X. Gao, D. Zhang, Y. Liu, G. Huang, Synthesis and thermal properties of modified room temperature vulcanized (rtv) silicone rubber using polyhedral oligomeric silsesquioxane (poss) as a cross linking agent, RSC Adv. 4 (2014) 41453-41460. 
[20] K. Madhavan, B. Reddy, Structuregas transport property relationships of poly(dimethylsiloxaneurethane) nanocomposite membranes, Journal of Membrane Science 342 (2009) 291 - 299.

[21] D. Chen, C. Huang, X. Hu, Preparation and characterization of novel polydimethylsiloxane composites used poss as cross-linker and fumed silica as reinforcing filler, Polymer Composites 34 (2013) 1041-1050.

[22] D. Chen, Y. Liu, H. Zhang, Y. Zhou, C. Huang, C. Xiong, Influence of polyhedral oligomeric silsesquioxanes (poss) on thermal and mechanical properties of polydimethylsiloxane (pdms) composites filled with fumed silica, Journal of Inorganic and Organometallic Polymers and Materials 23 (2013) 1375-1382.

[23] T. F. Baumann, T. V. Jones, T. Wilson, A. P. Saab, R. S. Maxwell, Synthesis and characterization of novel pdms nanocomposites using poss derivatives as cross-linking filler, Journal of Polymer Science Part A: Polymer Chemistry 47 (2009) 2589-2596.

[24] J. chao Huang, C. bin He, Y. Xiao, K. Y. Mya, J. Dai, Y. P. Siow, Polyimide/poss nanocomposites: interfacial interaction, thermal properties and mechanical properties, Polymer 44 (2003) 4491 - 4499.

[25] P. Marchetti, M. F. Jimenez Solomon, G. Szekely, A. G. Livingston, Molecular separation with organic solvent nanofiltration: A critical review, Chemical Reviews 114 (2014) 10735-10806. PMID: 25333504.

[26] W. Lau, A. Ismail, N. Misdan, M. Kassim, A recent progress in thin film composite membrane: A review, Desalination 287 (2012) 190 199. Special Issue in honour of Professor Takeshi Matsuura on his 75th Birthday.

[27] M. J. Raaijmakers, N. E. Benes, Current trends in interfacial polymerization chemistry, Progress in Polymer Science 63 (2016) 86 - 142.

[28] M. Dalwani, J. Zheng, M. Hempenius, M. J. T. Raaijmakers, C. M. Doherty, A. J. Hill, M. Wessling, N. E. Benes, Ultra-thin hybrid polyhedral silsesquioxane-polyamide films with potentially unlimited 2d dimensions, J. Mater. Chem. 22 (2012) 14835-14838.

[29] J. Duan, E. Litwiller, I. Pinnau, Preparation and water desalination properties of poss-polyamide nanocomposite reverse osmosis membranes, Journal of Membrane Science 473 (2015) 157 - 164.

[30] M. J. T. Raaijmakers, M. A. Hempenius, P. M. Schön, G. J. Vancso, A. Nijmeijer, M. Wessling, N. E. Benes, Sieving of hot gases by hyper-cross-linked nanoscale-hybrid membranes, Journal of the American Chemical Society 136 (2014) 330-335. PMID: 24308639.

[31] E. J. Kappert, M. J. Raaijmakers, W. Ogieglo, A. Nijmeijer, C. Huiskes, N. E. Benes, Temperature calibration procedure for thin film substrates for thermo-ellipsometric analysis using melting point standards, Thermochimica Acta 601 (2015) 29 - 32.

[32] M. J. T. Raaijmakers, E. J. Kappert, A. Nijmeijer, N. E. Benes, Thermal imidization kinetics of ultrathin films of hybrid poly(poss-imide)s, Macromolecules 48 (2015) 3031-3039.

[33] N. Stafie, D. Stamatialis, M. Wessling, Effect of $\{$ PDMS $\}$ cross-linking degree on the permeation performance of pan/pdms composite nanofiltration membranes, Separation and Purification Technology 45 (2005) 220 - 231.

[34] C. V. Rumens, M. A. Ziai, K. E. Belsey, J. C. Batchelor, S. J. Holder, Swelling of pdms networks in solvent vapours; applications for passive rfid wireless sensors, J. Mater. Chem. C 3 (2015) 10091-10098.

[35] J. N. Lee, C. Park, G. M. Whitesides, Solvent compatibility of poly(dimethylsiloxane)-based microfluidic devices, Analytical Chemistry 75 (2003) 6544-6554. PMID: 14640726.

[36] M. J. T. Raaijmakers, W. Ogieglo, M. Wiese, M. Wessling, A. Nijmeijer, N. E. Benes, Sorption Behavior of Compressed CO2 and CH4 on Ultrathin Hybrid Poly(POSS-imide) Layers, ACS Applied Materials \& Interfaces 7 (2015) 26977-26988. 